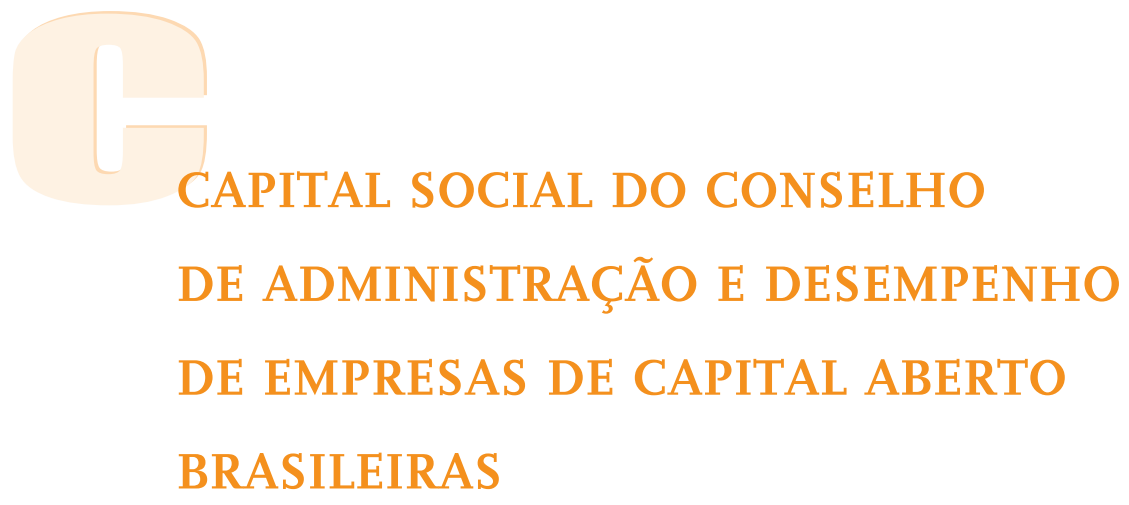

CEZAR EDUARDO ARAMHA

Bacharel em Informática pela Universidade Federal do Paraná (UFPR). Mestre em Administração pela Escola de Negócios da Universidade Positivo. Rua José Lopes dos Santos, 359, Santo Inácio, Curitiba - PR - Brasil - CEP 82300-250

E-mail: cezar.aranha@gmail.com

LUCIANO ROSSONI

Doutor em Administração pelo Centro de Pesquisa e Pós-Graduação em Administração da Universidade Federal do Paraná (UFPR). Professor do Programa de Pós-Graduação em Administração da Universidade do Grande Rio (Unigranrio). Pesquisador do Instituto Brasileiro de Estudos e Pesquisas Sociais (Ibepes). Rua da Lapa, 86, $9^{\circ}$ andar, Centro, Rio de Janeiro - RJ - Brasil - CEP 22220-040

E-mail: Irossoni@gmail.com

\title{
WESLEY MIEIDES-DA-SILVA
}

Livre-docente em Administração (Finanças) pela Faculdade de Economia, Administração e Contabilidade da Universidade de São Paulo (FEA-USP). Professor da Escola de Administração de Empresas de São Paulo da Fundação Getulio Vargas (FGV/SP).

Rua Itapeva, 474, $8^{\circ}$ andar, Bela Vista, São Paulo - SP - Brasil - CEP 01332-000

E-mail: mr.mendesdasilva@gmail.com

Este artigo pode ser copiado, distribuído, exibido, transmitido ou adaptado desde que citados, de forma clara e explícita, o nome da revista, a edição, o ano e as páginas nas quais o artigo foi publicado originalmente, mas sem sugerir que a RAM endosse a reutilização do artigo. Esse termo de licenciamento deve ser explicitado para os casos de reutilização ou distribuição para terceiros. Não é permitido o uso para fins comerciais. 


\section{RESUMO}

Objetivo: Buscamos analisar o efeito do capital social do conselho de administração (board) no valor de mercado das empresas listadas na BM\&F Bovespa, utilizando como indicador do capital social os recursos relacionais presentes nos laços diretos, indiretos e heterogêneos do board.

Originalidade/lacuna/relevância/implicações: Pioneiramente, nosso estudo aponta que as inconsistências dos resultados de estudos que avaliam redes de conselheiros muitas vezes ocorrem porque grande parte dos estudos considera somente a centralidade e a posição das companhias na rede. Com isso, desconsideram que as relações podem apresentar maior ou menor grau de capital social. Principais aspectos metodológicos: Avaliamos empiricamente as relações entre companhias a partir dos conselheiros e diretores que elas compartilham. Usando análise de redes sociais, identificamos quais são as relações diretas e indiretas e as lacunas estruturais das companhias, e também mensuramos o capital representado por cada relacionamento a partir do valor de mercado dessas relações, compondo nossa medida de recursos relacionais. Por fim, usando modelos econométricos, investigamos os efeitos dos recursos relacionais no valor de mercado. Síntese dos principais resultados: Nossos resultados apontaram que recursos relacionais heterogêneos têm influência mais forte e significativa do que recursos disponíveis nas relações diretas do board, enquanto recursos disponíveis nas relações indiretas não foram significativos.

Principais considerações/conclusões: Demonstramos que o capital social realmente importa, pois os recursos fornecidos pelos alters são recursos potenciais que podem ser usados em benefício de uma firma individualmente e também que as lacunas estruturais são meios para obter informações e recursos, possibilitando uma maior vantagem competitiva.

\section{PALAVRAS-CHAVE}

Capital social. Conselho de administração. Análise de redes sociais. Desempenho. Mercado de capitais. 


\section{INTRODUÇÃ O}

Há tempos, o mercado de capitais brasileiro é visto com um dos mais importantes do mundo. Como citam Mendes-da-Silva e Onusic (20I4), o volume de ações negociadas no Brasil passou de I,21\% do produto interno bruto (PIB), em I990, para 43,I5\%, em 20I0, configurando-se como o quarto maior do mundo em valor de mercado. Sua importância não é dada somente pelo volume de recursos, mas também porque países em desenvolvimento procuraram abrir seus mercados para receber investimentos internacionais, com o objetivo de desenvolver e modernizar suas economias (Weber, Davis, \& Lounsbury, 2009). Pode-se ainda identificar a importância do mercado de capitais para as economias emergentes a partir dos noticiários econômicos que usam os volumes de negócios e índices desses mercados como termômetro da economia do país e da economia global.

Tendo em vista a relevância dos mercados de capitais para a economia, um dos elementos que vêm ganhando destaque devido ao seu papel crucial na gestão das companhias listadas em bolsa é o conselho de administração, também denominado board (Johnson, Schnatterly, \& Hill, 20I3). Os conselhos de administração caracterizam-se por representarem os acionistas junto às companhias na definição de suas estratégias e na busca de seus objetivos visando à maximização dos rendimentos, além de funcionarem como peças de ratificação e de monitoramento (Lazzarini, 20II). Assim, espera-se que o conselheiro, em particular, e o conselho, em geral, sejam responsáveis por monitorar, decidir e aconselhar os executivos e acionistas, buscando a valorização dos ativos da companhia.

Mesmo havendo forte crença de que os conselhos de administração têm influência significativa e direta no desempenho financeiro das companhias (Rossoni \& Machado-da-Silva, 20Io), os resultados que suportam tal crença ainda são obscuros (He \& Huang, 20II). Como apontam He e Huang (20II), Johnson et al. (2013) e Stevenson e Radin (2009), pesquisas examinando a relação entre conselho e desempenho têm se concentrado em características formais e estruturais do board, no capital intelectual, no perfil demográfico dos conselheiros e no grau de independência do conselho, mas sem chegar a resultados consistentes. Como ainda pode ser visto na ampla revisão de Johnson et al. (2013), além de tais elementos, os estudos mais recentes aparentemente estão focando mais as relações que os conselheiros estabelecem fora da corporação.

Os conselhos de administração são formados por conselheiros que interagem entre si e também com conselheiros de outras empresas por meio de uma relação conhecida como board interlocking, em que um conselheiro ou diretor de uma empresa ocupa também uma cadeira no conselho de administração de 
outra empresa. Mas qual seria a importância dessas relações entre conselhos para as empresas de capital aberto ou quais seriam os benefícios de ter conselheiros com múltiplas relações? Davis (I996) e Mizruchi (I996) apontam que bons conselheiros tendem a participar de maior número de companhias, tal como de diferentes grupos, sendo a centralidade e a posição desses conselheiros na rede indício de seu prestígio. Além disso, se eles estão mais fortemente imersos em uma teia de relacionamentos, sofrem maior pressão social para que possam atuar de forma responsável (Davis, I996). Ademais, estudos apontam que conselheiros mais bem posicionados na rede tendem a ter maior capacidade de receber informação, recursos e conhecimento por meio do seu acesso privilegiado a diferentes grupos não conectados entre si (Davis, I996), ou seja, maior capital social (Burt, I992; Kim, 2007).

Apesar de tais argumentos em favor da maior centralidade e posição privilegiada do board serem convincentes, também há controvérsias sobre o fato de as relações afetarem positiva ou negativamente o desempenho da firma (Fracassi \& Tate, 20I2; Johnson et al., 2013). Há tanto resultados positivos, evidenciados em alguns estudos (por exemplo, Mendes-da-Silva, Rossoni, Martin, \& Martelanc, 2008; Mizruchi, Stearns, \& Marquis, 2006; Rossoni \& Machado-da-Silva, 2013), como negativos (Fracassi \& Tate, 2012; Santos \& Silveira, 2007). Todavia, esses estudos se ativeram somente a identificar os efeitos da posição privilegiada dos conselhos e de seus conselheiros, utilizando-se de vários indicadores de análise de redes sociais (por exemplo, centralidade de grau, poder de Bonacich, lacunas estruturais). Nesses estudos, deixaram-se de lado a quantidade, o tipo e a disponibilidade de recursos que esses conselheiros hipoteticamente trazem para as organizações.

Ora, se o capital social (capital disperso nas redes de relações) é composto tanto pelas relações entre atores sociais quanto pelos recursos que estão imersos em determinada rede de relações (Lin, 200I), é provável que seus efeitos não se resumam somente em se ter uma posição privilegiada. Dessa forma, estamos interessados em investigar a influência dos recursos imersos na rede no desempenho, o que chamamos aqui de recursos relacionais, propondo uma avaliação empírica do capital social presente nas relações entre os conselhos de administração por meio da interação de suas dimensões básicas: redes e recursos. Diante do exposto, objetivamos neste estudo avaliar o efeito do capital social do conselho de administração (board) no valor de mercado das empresas listadas na BM\&F Bovespa, nos anos de 20 Io e 20II. Para tanto, utilizamos como indicador do capital social os recursos relacionais presentes nos laços diretos, indiretos e heterogêneos do board. 


\section{CAPITAL SOCIAL DO BOARD \\ E DESEMPENHO: TEORIA E HIPÓTESES}

De forma geral, o capital social é visto como um bem social, em função das relações entre os atores, nos quais o capital social é capturado pelas trocas que ocorrem nas relações sociais (Lin, 200I). Em termos específicos, o capital social refere-se à ideia de que o investimento nas relações sociais pode ocasionar maior acesso à ampla variedade de recursos (Lin, 200I). Dessa forma, a conexão com diferentes redes ou grupos aumenta as chances de aquisição de vantagens (Burt, I992). Como ainda argumenta Nahapiet (2008, p. 580), "aqueles que fazem melhor fazem-no por meio das suas conexões e relacionamentos [...] eles são mais capazes de acessar um leque de oportunidades e recursos que afeta seu desempenho e beneficiar-se dele”. Em termos objetivos, Portes (I998, p. 7) pontua que "o capital econômico está nas contas bancárias das pessoas, o capital humano/cultural está dentro de suas cabeças e o capital social está na estrutura de seus relacionamentos".

Os mecanismos sociais que suportam as vantagens do capital social envolvem: maior facilidade no fluxo da informação, influência que os laços sociais exercem sobre os agentes que tomam decisões, acreditação e suporte social dado pelas relações, reforço da identidade e reconhecimento (Lin, 200I). Já suas dimensões envolvem o volume de capital social em função do tamanho da rede e do volume de capital (econômico, cultural e simbólico), possuídos pelo indivíduo conectado à rede (Bourdieu, I980).

Para Mizruchi (1996), ao referir-se ao capital social do board e aos seus relacionamentos, os estudos referentes ao interlocking e desempenho das empresas apresentam uma diversidade de resultados, nos quais alguns autores encontraram associações positivas, outras negativas, mas em grau leve. Isso foi corroborado por revisão recente de Johnson et al. (2013), que destacam a complexidade e o caráter contingencial do fenômeno. Em comum entre eles, estão as formas como o capital social tende a ser operacionalizado.

Por exemplo, Davis e Mizruchi (I999) desenvolveram estudo para entender como a reestruturação da indústria nos Estados Unidos afetou o posicionamento dos bancos na rede. Para corroborar suas hipóteses de estudo, esses autores utilizaram os conceitos da centralidade de Freeman e Bonacich para determinar a posição dessas empresas. Já Weber et al. (2009) investigaram as causas da adoção de políticas de bolsas de valores nos países em desenvolvimento e adotaram o indicador de centralidade do país no sistema econômico mundial como fator explicativo da adoção de bolsas de valores. Mendes-da-Silva et al. (2008), por sua vez, verificaram relação significativa de uma posição privilegiada (centralidade, 
densidade e coesão) do conselho de administração de empresas do novo mercado da Bovespa no valor de mercado e no endividamento de companhias listadas. Posteriormente, Mendes-da-Silva (20II) investigou a existência de associações entre o posicionamento da firma na rede de relações e o valor das empresas. Para isso, utilizou-se do conceito de centralidade (degree normalizado, betweenness normalizado e eingenvector normalizado), encontrando evidências de que, em relação ao valor das empresas, há níveis ótimos de centralidades.

Com objeto um pouco diferenciado, Pusser, Slaughter e Thomas (2006) analisaram os patrocinadores de faculdades de ensino superior nos Estados Unidos, descrevendo o interlock entre conselhos de administração das universidades e conselhos de administração das empresas americanas. Um dos indicadores utilizados para determinar a posição dos conselhos foi a centralidade de grau (degree), em que os autores concluíram que membros dos conselhos das universidades privadas são mais propensos a se alinhar com a tecnociência da nova economia, enquanto os membros dos conselhos das universidades públicas são mais propensos a se alinhar às corporações financeiras. Por fim, em revisão recente, Johnson et al. (2013) sumarizam os resultados acerca do capital social do conselho como equivocados, propondo que esse tipo de capital não é independente das demais condições.

Apesar desses resultados contraditórios, ainda se insiste em que as relações entre conselhos podem ajudar a explicar a efetividade das companhias abertas, especialmente porque, mesmo havendo boa quantidade de estudos, o tema ainda não se esgotou. Em especial, acreditamos que esses estudos não deram atenção devida a um dos elementos do capital social: os recursos existentes nas relações sociais.

Assim, como Lin (200I) advoga, o capital social está presente nas trocas que decorrem das relações entre atores, por meio de recursos mobilizados e disponíveis nessas relações. Ou seja, se o capital social se configura por recursos e relações, não faz sentido dar atenção a somente uma dimensão, desconsiderando outra. Assim, propõe-se que o acesso a recursos inseridos em redes sociais e a utilização deles podem levar a melhores condições socioeconômicas para atores sociais, em especial companhias de capital aberto, em que quanto maior for o capital potencialmente presente nas relações sociais, maiores serão as chances de elas tomarem boas decisões e serem julgadas mais confiáveis e legítimas. Se isso importa para os investidores, é provável que tais recursos relacionais tenham associação positiva com o valor de mercado. Mais ainda, sabendo que alguns desses recursos são abstratos e fluidos, como a informação e a influência, acreditamos que as relações indiretas também possam ser potenciais catalisadoras, podendo ter implicações no valor das companhias abertas. Portanto, a partir desses argumentos, foram derivadas as seguintes hipóteses: 
- Hipótese ra: Quanto maior a quantidade de recursos disponíveis nas relações diretas, maior é o valor de mercado da companhia.

- Hipótese rb: Quanto maior a quantidade de recursos disponíveis nas relações indiretas, maior é o valor de mercado da companhia.

Mas será que as relações do board impactam da mesma forma? Será que não somente o volume desses recursos relacionais importa, mas também a heterogeneidade deles? De acordo com Burt (1992), as lacunas estruturais - ou laços não redundantes - são oportunidades para intermediar o fluxo de informação entre atores que estão em lados opostos, em que as ligações fracas entre esses atores da rede são lacunas que podem ser mais bem trabalhadas, de modo a obter maiores recursos.

Esses recursos, por estarem fragmentados na rede de relações, têm maior possibilidade de possuírem informações diversas e heterogêneas. Segundo alguns autores (Rossoni \& Machado-da-Silva, 20I3; Uzzi \& Spiro, 2005), esse somatório de características traz maior possibilidade de obter vantagem competitiva. Vale reforçar que as lacunas não significam que os atores não estão conectados, mas que eles têm maior possibilidade de acessar conteúdo informacional não redundante, porque seus laços ocorrem entre atores não diretamente conectados ou que fazem parte de agrupamentos distintos.

A redundância de informações, por sua vez, pode ocorrer de duas formas (Burt, 1992): por contatos diretos (coesão), em que dois contatos são considerados redundantes, na extensão que apresentam relações fortes e coesas; e por contatos indiretos (equivalência estrutural), em que dois contatos são considerados estruturalmente equivalentes, à medida que apresentam as mesmas relações, mesmo que não estejam diretamente ligados. Assim, para os recursos serem considerados heterogêneos, eles não devem ser redundantes por nenhuma dessas formas.

No que se refere aos conselhos de administração, alguns estudos tendem a se aproximar dessa lógica. Kim (2007) analisou os efeitos da proporção de conselheiros externos e o seu capital social no valor da empresa, e chegou à conclusão de que o board tem capacidade de extrair recursos valiosos a partir do ambiente e que isso é uma função do capital social dos conselheiros. Os resultados desse estudo também sugerem que um conselho de administração eficaz não surge sem uma rede externa eficaz dos membros do conselho (interlocking). Já Rossoni e Machado-da-Silva (2013) avaliaram como as diferentes origens da legitimidade organizacional condicionaram o desempenho das empresas listadas na BM\&F Bovespa, a partir do valor de mercado. Os autores utilizaram a ideia de lacunas estruturais para testar a hipótese de quanto maior a proporção 
de lacunas estruturais do conselho administrativo, maior o valor de mercado. Os resultados do estudo citado corroboraram essa hipótese, em que conselhos com laços mais heterogêneos têm maior valor de mercado. Por fim, Uzzi e Spiro (2005) analisaram a estrutura de redes small words dos artistas que fizeram musicais da Broadway de I945 a I989. Esses autores utilizaram a intuição de laços fracos (Granovetter, I973) e lacunas estruturais (Burt, I992), e constataram relação significativa entre tais laços com a capacidade de peças de teatro em atingir sucesso de crítica e público. Assim, perante tais resultados, esperamos neste estudo que a heterogeneidade dos laços condicione o efeito dos recursos relacionais do board no valor de mercado, o que leva à seguinte hipótese:

- Hipótese 2: Quanto maior a heterogeneidade de recursos disponível nas relações diretas, maior é o valor de mercado da empresa.

\section{MÉTODO}

\subsection{DADOS E AMOSTRA}

A população deste estudo compreende as companhias listadas na BM\&F Bovespa nos anos de 2010 e 20II. Foram selecionadas aquelas companhias com liquidez das ações maior que o,or\%, já que alguns indicadores do estudo só são possíveis de ser avaliados em firmas com negociação efetiva em bolsa. A partir da identificação de firmas no cadastro de emissoras ativas da Comissão de Valores Mobiliários (CVM), foram cruzados os dados com as informações financeiras da base Economatica ${ }^{\circledR}$, em que obtivemos 334 casos em 20 Io e 350 em 20II. Além disso, como o valor de mercado foi coletado considerando $t+\mathrm{I}$ ano, conseguimos informações de 3 IO empresas em $201 \mathrm{II}$ e 307 empresas em 2012.

Por fim, consolidando os dados financeiros obtidos por meio da base de dados Economatica ${ }^{\circledR}$ com as demais fontes de dados cadastrais (sistema de divulgação externa ITR, DFP, IAN, IEP, FC, FR da CVM; informações da BM\&F Bovespa; formulários de referência das companhias), nossa amostra se resumiu a 513 casos válidos, oriundos de 283 firmas, organizados em painel não balanceado.

\section{REDES DE CONSELHOS E DE PROPRIEDADE CRUZADA}

Montamos a rede de conselhos de empresas a partir de informações disponíveis nos formulários de referência das companhias abertas identificadas no 
sistema da CVM. Primeiro, listamos todas as firmas e seus respectivos conselheiros e diretores individualmente para os anos de 20 Io e $201 \mathrm{I}$, o que permitiu que gerássemos uma rede de incidência no formato 2-mode (empresas versus conselheiros) para cada ano. Depois, por meio do software Pajek, criamos as redes de relações entre conselhos de administração (formato 1-mode), em que duas firmas eram diretamente ligadas se compartilhavam pelo menos um conselheiro ou diretor, fenômeno conhecido como board interlocking. Exportaram-se esses dados das redes de conselhos para o software Ucinet, utilizado para gerar os indicadores relacionais no nível da firma, que foram posteriormente incorporados no painel. Já para a criação das redes de empresas de propriedade cruzada, utilizou-se essa mesma sequência, porém consideramos que duas empresas estariam conectadas se apresentassem os mesmos proprietários na listagem de acionistas disponível no sistema da CVM ou se uma delas apresentasse ações da outra.

\subsection{VARIÁVEL DEPENDENTE}

\subsubsection{Desempenho organizacional}

Com base em estudos anteriores (Bhagat \& Bolton, 2008; Black, Carvalho, \& Gorga, 20I2; Black \& Kim, 20I2; Carvalhal-Da-Silva \& Leal, 2005; Kim, 2007; Mendes-da-Silva et al., 2008; Rossoni \& Machado-da-Silva, 2013; Silveira, Barros, \& Famá, 2006), definiu-se como indicador de desempenho de empresas listadas em bolsa o valor de mercado, que foi avaliado por meio do $Q$ de Tobin, que é a soma do valor de mercado das ações, acrescido da soma de seus débitos, sendo este valor dividido pelo valor contábil do ativo (Bozec, Dia, \& Bozec, 20ıо; Chung \& Pruitt, I994). Essa medida foi operacionalizada para os anos de 20 II e 20I2, seguindo a adaptação de Chung e Pruitt (I994, p. 72), formalmente definida como:

$$
Q \text { de Tobi }=\frac{V M a O+V M a P+V C D c l P}{V C A T}
$$

em que $\mathrm{VMaO}=$ valor de mercado das ações ordinárias; $\mathrm{VMaP}=$ valor de mercado das ações preferenciais; $V C D c l P=$ valor contábil das dívidas de curto e longo prazos da empresa; $V C A T$ = valor contábil do ativo total da organização (Chung \& Pruitt, 1994). 


\subsection{VARIÁVEIS INDEPENDENTES}

\subsubsection{Capital social do board (conselho de administração)}

A operacionalização do capital social foi dada pela soma dos recursos relacionais presentes em três tipos de relação: direta, indireta e heterogênea.

Assim, para se chegar ao capital social das relações diretas, primeiramente foram identificados, em cada ano, os laços diretos que cada firma possuía com as demais por meio das redes de conselhos de administração, o que é conhecido no vocabulário de análise de redes sociais como egonetwork. Após isso, identificamos o valor de mercado (valor total das ações negociadas em bolsa) de cada uma das relações da firma, que é o que denominamos recursos relacionais. Por fim, somou-se o valor desses recursos relacionais de cada laço, o que gerou nosso indicador de capital social das relações diretas da empresa.

Já o capital social das relações indiretas de cada firma, como o próprio nome diz, foi obtido por meio da soma dos recursos relacionais (valor de mercado) das relações indiretas de primeiro grau. Apesar de similar ao procedimento anterior, para se chegar às relações indiretas, houve a necessidade de realizar o seguinte procedimento: a partir das redes de relações diretas, foi calculada a distância geodésica entre as firmas, salvando-a em uma matriz de distâncias; depois, recodificamos essa rede de forma que as relações indiretas de primeiro grau (valor 2 na rede) fossem identificadas por meio de um código binário com valor I, enquanto todas as outras distâncias foram codificadas como zero. Com isso, foi possível identificar, para cada nó (firma), quais eram suas relações indiretas de primeiro grau.

Por fim, o capital social das relações heterogêneas foi gerado da seguinte forma:

- Executou-se o procedimento structural holes no Ucinet, e foi salva a matriz DR (dyadic redundancy), que apresenta o grau de redundância de cada alter (relações diretas) em relação a cada um dos egos (firma) da rede. Resumidamente, a redundância indica o percentual de laços que o ego e o alter possuem em uma rede ego, em que quanto maior o valor, mais redundante o laço. Para obter mais detalhes, ver Burt (I992).

- Subtraiu-se o escore de redundância de cada alter válido por um, obtendo-se, assim, um escore de heterogeneidade dos alters, que foi tabulado em novas matrizes.

- Multiplicou-se o valor de mercado de cada relação existente (alter) pelo seu respectivo escore de heterogeneidade. 
- Somou-se o produto das relações de cada firma, tendo assim o capital social das relações heterogêneas da empresa.

Como análise adicional, também foi avaliado o efeito da posição do conselho de administração em relação ao conselho das demais empresas. Para tanto, utilizamos três indicadores já contemplados em estudos anteriores (Fracassi \& Tate, 2012; Johnson et al., 2013; Kim, 2005; Mendes-da-Silva et al., 2008; Mendes-da-Silva, 20II; Rossoni \& Machado-da-Silva, 20I3):

I. Centralidade de grau: essa medida de centralidade reflete o número de laços adjacentes de um ator na rede (Freeman, I979; Freeman, Borgatti, \& White, I99I) que, no nosso estudo, remete ao número de empresas ligadas pelos conselheiros ou diretores que determinada firma compartilha com as demais.

2. Eigenvector: trata-se da centralidade alpha de Bonacich, que avalia o grau de centralização de um nó (nesse caso, a empresa), considerando também a centralidade dos laços vizinhos, ou seja, ela leva em conta o grau de hierarquização das relações para compor o indicador (De Nooy, Mrvar, \& Batagelj, 2005; Hanneman \& Riddle, 2005). Vale lembrar que essa medida se diferencia da centralidade de grau, que considera somente o número de laços adjacentes, por levar em conta também a centralidade de tais laços.

3. Lacunas estruturais: para a operacionalização das lacunas estruturais (structural holes), utilizamos a medida de eficiência dos laços (Burt, I992), que mensura o número de contatos não redundantes EffSize em relação ao total de contatos $n$ de um ator $i$. Como se trabalhou com dados binários, utilizou-se a forma simplificada da equação desenvolvida por Borgatti (I997). Formalmente, considerando que um ator $i$ apresenta $n$ número de contatos, pode-se avaliar o número de contatos redundantes por meio da equação $D$ alters $=2 l / n$, em que $l$ é o número de laços entre $n$ (alters). Já que $D$ alters indica o total de laços redundantes, consideramos como laços não redundantes EffSize como $n-D$ alters. Dessa forma, a proporção de laços não redundantes Efficiency é dada por EffSize/n. Como há variação anual de conselheiros que fazem parte das organizações, calculou-se essa medida para cada ano de participação da empresa na bolsa.

Para ilustrar as relações entre conselhos de administração, recortamos a porção mais central da rede na Figura I $(\mathrm{K}$-core $=5)$. Uma delas, a Empresa Brasileira de Aeronáutica (Embraer), foi a empresa com maior quantidade de recursos relacionais (290 bilhões). 


\section{FIGURA I}

\section{CENT'RO DA REDE DE RELAÇÕES}

ENTRE COMPANHIAS (K-CORE = 5)

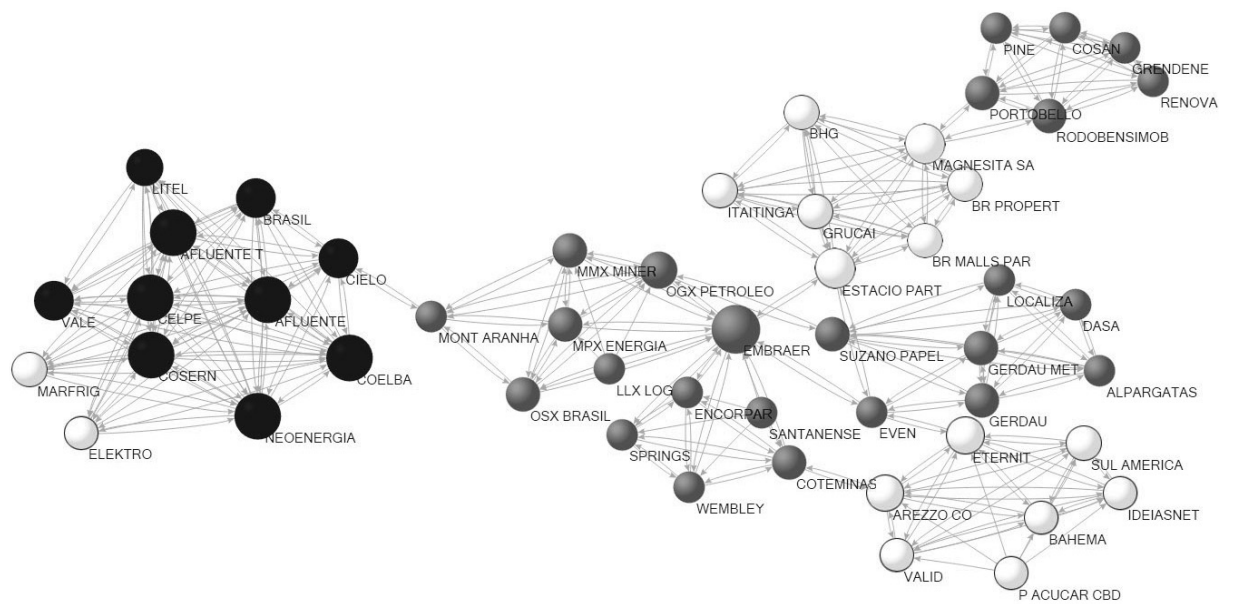

Nós brancos: K-core = 5; cinzas: K-core= 6; pretos: K-core = 7. Dados de 2010 .

Fonte: Elaborada pelos autores.

\subsection{VARIÁVEIS DE CONTROLE}

\subsubsection{Capital social da propriedade cruzada}

Com o objetivo de ter uma hipótese concorrente à do capital social do conselho de administração, criou-se um controle que considera o capital social oriundo do entrelaçamento entre proprietários. Assim, duas firmas apresentam propriedade cruzada quando pelo menos uma delas tem participação acionária na outra (Aldrighi \& Mazzer, 2007). Para tanto, para se chegar ao capital social das relações de propriedade, para cada uma das companhias, foram identificadas aquelas que tinham proprietários em comum ou aquelas em que uma fosse acionista da outra. Depois, identificou-se o valor de mercado (valor total das ações negociadas em bolsa) de cada uma dessas empresas. Por fim, foi somado o valor de mercado de cada uma delas, formando o indicador de capital social das relações de propriedade.

\subsubsection{Idade}

Como fizeram outros estudos (Haniffa \& Cooke, 2002; Mendes-da-Silva, Ferraz-Andrade, Famá, \& Maluf, 2009), a idade da empresa foi operacionalizada 
por meio do logaritmo natural do tempo de atuação no mercado de ações brasileiro, mais especificamente na BM\&F Bovespa.

\subsubsection{Tamanho da empresa}

Utilizamos o valor contábil do ativo total como proxy do tamanho da firma. Tal informação foi coletada por meio da base Economatica ${ }^{\circledR}$. Ademais, com o objetivo de diminuir problemas de simetria e curtose, logaritmizou-se o tamanho da empresa, como também foi feito em outros estudos (Mendes-da-Silva et al., 2008; Rossoni \& Machado-da-Silva, 2013; Silveira et al., 2006).

\subsubsection{Ano}

Visando evitar problemas relacionados à sazonalidade, tal como tendências temporais, controlamos o efeito do tempo por meio de variáveis dummy, o que é prática comum em dados em painel (ver, por exemplo, Carvalhal-Da-Silva \& Leal, 2005; Procianoy \& Verdi, 2009; Silveira, Leal, Carvalhal-Da-Silva, \& Barros, 20Io). Assim, o ano de 20 Io foi considerado como categoria de referência, e, no modelo, identificou-se o ano de 20 II por meio de uma variável dummy.

\subsubsection{Setor}

Controlou-se o efeito do setor, já que existe evidência de que ele é antecedente do valor de mercado (Black et al., 2012; Procianoy \& Verdi, 2009; Silveira et al., 2006, 2010). Para tanto, foram criadas s-1 variáveis dummy, em que $s$ é o número de setores identificados na Economatica ${ }^{\circledR}$, considerando como categoria de referência o setor "outros" por este apresentar maior número de observações.

Vale destacar que foram excluídas algumas variáveis de controle, como listagens em mercados diferenciados e tamanho do conselho, em razão de problemas de colinearidade, especialmente pelo fato de os painéis terem sido executados com efeitos fixos.

\section{PROCEDIMENTO DE ANÁLISE}

Foi analisada a influência do capital social do board no valor de mercado a partir da análise de dados em painel, cujas variáveis foram hierarquicamente incorporadas em sete modelos distintos. Optou-se pelo modelo econométrico 
em painel porque havia vários casos $(N)$ com um número de observações no tempo (T), configurando NxT observações. Para tanto, foram testadas três alternativas para avaliar qual delas era a mais adequada: I. agrupamento de dados de corte a partir dos mínimos quadrados ordinários (MQO agrupado), 2. os efeitos fixos (EF) e 3. efeitos aleatórios (EA).

Segundo Greene (2000), a escolha do modelo mais ajustado depende da confrontação de três testes de hipóteses: I. a existência ou não de um único intercepto das unidades de corte transversal (avaliada por meio do teste de $\mathrm{F}$ de Chow); 2. se a variância do intercepto é igual a zero (teste multiplicador de Lagrange modificado pela proposição de Breusch e Pagan); 3. se os estimadores são consistentes a partir da estimação dos mínimos quadrados generalizados (teste de Hausman). A partir da avaliação dessas hipóteses, escolheu-se o melhor modelo para cada uma das relações entre variáveis. Em seguida, buscou-se maior robustez de resultados por seis formas diferentes:

- Avaliamos se a variável dependente apresentava problemas de distribuição graves.

- Verificamos se as variáveis independentes apresentavam a forma funcional linear em relação à variável dependente.

- Avaliamos os modelos com relação a problemas de heterocedasticidade por meio do teste de White. Caso houvesse, tais problemas seriam tratados com o uso de erros padrão robustos.

- Verificamos se havia problemas de colinearidade entre as variáveis independentes. Como o modelo com todas as variáveis (modelo 7) apresentou problemas de multicolinearidade (tolerância menor que o,2 e VIF maior que 5), a análise das hipóteses foi feita por meio dos modelos que apresentavam cada variável independente individualmente.

- Ponderamos se os resultados se mantinham consistentes quando foi feita a regressão sem as variáveis não significativas. Verificamos também se eles apresentavam a mesma tendência sem os outliers.

- No que tange à endogeneidade, não utilizamos variáveis instrumentais ou equações simultâneas (ver, por exemplo, Black \& Kim, 20I2; Silveira et al., 20Io), pois não há evidências claras na literatura acerca da endogeneidade de variáveis relacionais (Johnson et al., 2013; Mizruchi, I996).

\section{RESULTADOS}

A Tabela I apresenta os efeitos das variáveis no valor de mercado $(Q$ de Tobin). Como pode ser visto na tabela, após os testes de ajuste dos dados em painel de Chow, Breush-Pagan e Hausman, considerou-se o modelo fixo como o 
mais adequado para todos os modelos. Nessa linha de raciocínio, o uso do erro padrão robusto mostrou-se necessário, já que o teste de White apontou proble-

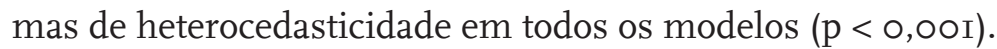

No modelo I, tem-se somente o efeito das variáveis de controle no $Q$ de Tobin, em que se omitiram os coeficientes do ano e do setor. Assim, das variáveis de controle, somente a idade da empresa foi significativa $(\mathrm{p}<\mathrm{0}, \mathrm{OI})$; isso quer dizer que, isoladamente, o incremento de um ano de permanência em bolsa está associado a um aumento de 0,53 ponto $(\ln (\mathrm{I}, 709))$ no $Q$ de Tobin. Isso demonstra que empresas com maior tempo de ações em bolsa tendem a ser mais valorizadas que as demais.

No modelo 2, como análise adicional, foram acrescentados três indicadores de posição do conselho na rede (centralidade de grau, eigenvector e lacunas estruturais). Mesmo havendo problemas de colinearidade, não houve distorções no sinal e na significância do efeito de cada variável individualmente. Não houve evidências de que o maior número de laços (centralidade de grau) com outras empresas por meio do compartilhamento de conselheiros e diretores estivesse associado a maior valor de mercado, tal como não foi observada influência significativa do percentual de laços fracos do conselho (lacunas estruturais).

No entanto, a centralidade de eigenvector ou poder de Bonacich, que considera não somente a centralidade da firma, mas também a centralidade de suas relações adjacentes, mostrou-se negativamente associada ao valor de mercado $(\mathrm{p}<\mathrm{O}, \mathrm{I})$. Assim, os resultados sugerem que aquelas empresas que apresentam menor valor de mercado tendem a se relacionar com empresas que apresentam conselhos de administração mais centrais.

Já no modelo 3, acrescentamos outra variável de controle: capital social da propriedade cruzada. Os resultados apontam que não há relação significativa entre ter relações de propriedade com empresas de maior valor de mercado e o $Q$ de Tobin.

A hipótese ra foi testada no modelo 4, em que a significância no nível de $90 \%$ dá indícios de que existe relação entre o capital social disponível nas relações diretamente estabelecidas pelo board e o valor de mercado. Assim, mesmo tal variável sendo pouco significativa e não apresentando significância no modelo 7 , entende-se que tal hipótese foi corroborada. Como efeito marginal do capital social das relações diretas no valor de mercado da empresa, foi possível perceber que, para cada Ioo bilhões a mais no capital social da empresa, há um aumento de I,2I ponto no $Q$ de Tobin.

Apesar de parecer um valor alto e pouco plausível de alcançar como capital social, vale destacar que a média do capital social das relações diretas é de I6 bilhões. Há casos, como o da Embraer, cujo capital social foi de 290 bilhões em 2011 . 
Porém, a hipótese ib, que foi testada no modelo 5, teve que ser refutada, já que não se conseguiu identificar relação significativa entre capital social disponível por meio de relações indiretas e o valor de mercado.

Por fim, a hipótese 2 foi corroborada no modelo 6 , cujos dados apontam para uma influência significativa e positiva do capital social das relações heterogêneas do board no $Q$ de Tobin $(\mathrm{p}<0,05)$. Esse modelo demonstra que, para cada Ioo bilhões de capital social ponderado pela heterogeneidade dos laços do conselho, há um incremento de I,909 ponto no $Q$ de Tobin.

Assim, quando se comparou o efeito marginal do capital social oriundo das relações heterogêneas com o das relações diretas, verificou-se que o primeiro, além de ser mais significativo, também apresenta maior amplitude do que o segundo.

Esse indício de maior poder de explicação das relações heterogêneas sobre as relações diretas também pode ser visto no modelo 7, que expõe todas as variáveis em conjunto. Como visto, quando todas as variáveis independentes concorreram entre si, somente o efeito do capital social das relações heterogêneas do conselho no valor de mercado foi significativo.

\section{TABELA I}

INFLUENCIA DO CAPITAL SOCIAL NO VALOR DE MERCADO ( $Q$ DE TOBIN)

\begin{tabular}{|c|c|c|c|c|c|c|c|}
\hline & MODELO 1 & MODELO 2 & MODELO 3 & MODELO 4 & MODELO 5 & MODELO 6 & MODELO 7 \\
\hline $\begin{array}{l}\text { Capital social do board: } \\
\text { relaçōes diretas }^{\mathrm{a}}\end{array}$ & & & & $\begin{array}{l}1,217^{*} \\
(0,658)\end{array}$ & & & $\begin{array}{l}-1,904 \\
(1,227)\end{array}$ \\
\hline $\begin{array}{l}\text { Capital social do board: } \\
\text { relação indiretas }\end{array}$ & & & & & $\begin{array}{l}0,368 \\
(0,360)\end{array}$ & & $\begin{array}{l}0,486 \\
(0,384)\end{array}$ \\
\hline $\begin{array}{l}\text { Capital social do board: } \\
\text { relações heterogêneas }\end{array}$ & & & & & & $\begin{array}{c}1,909 * * \\
(0,895)\end{array}$ & $\begin{array}{r}4,280^{* *} \\
(2,085)\end{array}$ \\
\hline $\begin{array}{l}\text { Capital social: propriedade } \\
\text { cruzada }^{\text {a }}\end{array}$ & & & $\begin{array}{l}0,552 \\
(0,394)\end{array}$ & & & & $\begin{array}{l}0,765 \\
(0,469)\end{array}$ \\
\hline Centralidade de grau & & $\begin{array}{l}0,068 \\
(0,079)\end{array}$ & & & & & \\
\hline Eigenvector & & $\begin{array}{c}-16,357^{*} \\
(8,736)\end{array}$ & & & & & \\
\hline Lacunas estruturais & & $\begin{array}{l}-0,096 \\
(0,125)\end{array}$ & & & & & \\
\hline
\end{tabular}

(continua) 
TABELA I (CONCLUSÃo)

INFLUENCIA DO CAPITAL SOCIAL NO VALOR

DE MERCADO ( $Q$ DE TOBIN)

\begin{tabular}{|c|c|c|c|c|c|c|c|}
\hline & MODELO 1 & MODELO 2 & MODELO 3 & MODELO 4 & MODELO 5 & MODELO 6 & MODELO 7 \\
\hline \multicolumn{8}{|c|}{ VARIÁVEIS DE CONTROLE } \\
\hline Idade da empresa (In) & $\begin{array}{c}1,709 * * * \\
(0,638)\end{array}$ & $\begin{array}{c}1,624 * * * \\
(0,621)\end{array}$ & $\begin{array}{l}1,227 * * \\
(0,614)\end{array}$ & $\begin{array}{c}1,198 * * \\
(0,606)\end{array}$ & $\begin{array}{c}1,191 * * \\
(0,601)\end{array}$ & $\begin{array}{l}1,194^{*} \\
(0,606)\end{array}$ & $\begin{array}{l}1,214 * * \\
(0,613)\end{array}$ \\
\hline Tamanho (In do ativo) & $\begin{array}{c}0,031 \\
(0,030)\end{array}$ & $\begin{array}{c}0,033 \\
(0,030)\end{array}$ & $\begin{array}{c}0,886 \\
(0,606)\end{array}$ & $\begin{array}{c}0,903 \\
(0,610)\end{array}$ & $\begin{array}{c}0,880 \\
(0,607)\end{array}$ & $\begin{array}{c}0,907 \\
(0,611)\end{array}$ & $\begin{array}{c}0,895 \\
(0,611)\end{array}$ \\
\hline Constante & $\begin{array}{l}-2,987 \\
(1,702)\end{array}$ & $\begin{array}{l}-2,634 \\
(1,623)\end{array}$ & $\begin{array}{r}-14,222 \\
(8,949)\end{array}$ & $\begin{array}{c}-14,400 \\
(9,011)\end{array}$ & $\begin{array}{r}-14,061 \\
(8,949)\end{array}$ & $\begin{array}{c}-14,448 \\
(9,012)\end{array}$ & $\begin{array}{r}-14,385 \\
(9,014)\end{array}$ \\
\hline Teste de White & $61,448 * * *$ & $64,849 * * *$ & $78,179 * * *$ & $78,563^{* * *}$ & $79,383 * * *$ & $78,842 * * *$ & $81,122 * * *$ \\
\hline Tesde F de Chow & $49,332 * * *$ & $48,640 * * *$ & $49,277 * * *$ & $49,327^{* * *}$ & $49,164^{* * *}$ & $49,312^{* * *}$ & $48,495^{* * *}$ \\
\hline Teste de Breusch-Pagan & $254,32 * * *$ & $251,97 * * *$ & $258,60 * * *$ & $258,99 * * *$ & $258,78^{* * *}$ & $258,79 * * *$ & $258,37 * * *$ \\
\hline Teste de Hausman & $23,444 * * *$ & $25,539 * * *$ & $13,941 * * *$ & $13,938 * * *$ & $14,473 * * *$ & $14,123 * * *$ & 14,622 ** \\
\hline Modelo & Fixo & Fixo & Fixo & Fixo & Fixo & Fixo & Fixo \\
\hline Número de casos & 513 & 513 & 501 & 501 & 501 & 501 & 501 \\
\hline Empresas & 283 & 283 & 282 & 282 & 282 & 282 & 282 \\
\hline Critério de Akaike & 1890,96 & 1894,79 & 1837,03 & 1836,89 & 1837,05 & 1836,71 & 1842,06 \\
\hline $\mathrm{F}$ & $53,53 * * *$ & $52,50 * * *$ & $54,29 * * *$ & $54,30 * * *$ & $54,28 * * *$ & $54,32 * * *$ & $53,08^{* * *}$ \\
\hline $\mathrm{R}^{2}$ & 0,985 & 0,985 & 0,986 & 0,986 & 0,986 & 0,986 & 0,986 \\
\hline $\mathrm{R}^{2}$ ajustado & 0,967 & 0,967 & 0,968 & 0,968 & 0,968 & 0,968 & 0,968 \\
\hline
\end{tabular}

Nota: Erro padrão entre parênteses. Dummies dos setores e dos anos ocultadas. ${ }^{a}$ Valores em bilhões (R\$).

$* \mathrm{p}<0, \mathrm{I}, * * \mathrm{p}<0,05 \mathrm{e}^{* * * *} \mathrm{p}<0,0$ I.

Fonte: Elaborada pelos autores.

\section{DIsCUSSÃO E CONCLUSÃo}

Neste estudo, buscou-se avaliar o efeito do capital social do conselho de administração (board) no valor de mercado das empresas listadas na BM\&F Bovespa. Para tanto, foram utilizados como indicadores do capital social os recursos relacionais presentes nos laços diretos, indiretos e heterogêneos do board. Como se 
afirmou na "Introdução", o mercado de capitais, por causa de sua regulamentação e das práticas de governança corporativa, tem se tornado uma área fértil no fornecimento de dados para pesquisas, envolvendo o board interlocking em função de suas características, principalmente no que se refere ao estudo econômico-financeiro e da rede de relações pessoais e corporativas que o mercado de capital envolve. Assim, ressaltam-se alguns aspectos identificados nesta pesquisa, antes de serem apresentadas as conclusões.

Em relação às normas de governança, no período deste estudo, $25 \%$ dos conselheiros do conselho de administração eram independentes, enquanto outros $75 \%$ foram indicados pelo controlador. Como não foi feita a análise identificada pelo nível de governança, seria imprudente qualquer análise referente a esse número. No entanto, isso destaca que a independência do conselho é algo ainda distante da realidade brasileira.

Outra informação a respeito do conselho de administração refere-se ao interlocking de conselheiros, cuja norma (Instituto Brasileiro de Governança Corporativa, 2009) sugere um número máximo de três interlocks. Em contraposição à norma, foi encontrado número muito superior a esse, por exemplo, no caso em que um único indivíduo participou de I5 conselhos ao mesmo tempo.

Nossos dados também possibilitaram verificar que as instituições bancárias privadas no Brasil não são centrais em relação à rede de interlocking. Para o ano de 20I0, o Brasil não apresentou nenhum banco entre as dez empresas mais centrais, enquanto, para o ano de 20II, apresentou somente um banco entre as dez primeiras empresas mais centrais. Isso diverge da estrutura relacional evidenciada por Davis e Mizruchi (I999), que desenvolveram pesquisa sobre o posicionamento das instituições bancárias americanas na década de I980.

Considerando os efeitos dos indicadores posicionais na rede no valor de mercado, apesar de haver resultados positivos evidenciados na literatura, inclusive no Brasil, os resultados reforçam os equívocos e a falta de consistência apontados pela revisão de Johnson et al. (2013). Por exemplo, enquanto os estudos de Mendes-da-Silva (20II) e Rossoni e Machado-da-Silva (20I3) encontraram resultados positivos com dados de até 2008 , em nosso estudo, com dados de 2010 a 20I2, as evidências são diferentes. Tanto que um dos indicadores que levantamos apresentou efeito negativo.

Uma possível explicação para o efeito negativo da centralidade eigenvector no valor de mercado pode ser visto em Black et al. (20I2). Conforme os autores, em mercados emergentes, como os países do Brics (Brasil, Rússia, Índia, China e África do Sul), companhias que estejam passando por dificuldades, com baixa valorização, podem buscar se fortalecer ou justificar mudanças tendo laços com empresas de maior valor de mercado, simplesmente por contratarem conselheiros ou diretores de tais companhias. 
Em relação à rede de propriedades, este estudo corrobora a pesquisa de Lazzarini (2007), na qual o autor identifica que, apesar da reestruturação que ocorreu no Brasil entre I995 e 2003 (privatizações e entrada de capitais estrangeiros), a estrutura de propriedade no país continua com a mesma característica: acionistas com fundos de pensões estatais, governos federais e estaduais. Talvez seja por essas características que não foi encontrada relação significativa entre capital social oriundo das relações de propriedade e o valor de mercado.

Por fim, em relação ao capital social do board, este estudo aponta que os recursos presentes nas relações diretas e heterogêneas afetam positiva e significativamente o valor de mercado das companhias abertas listadas na BM\&F Bovespa, demonstrando que quanto maior for o capital social do board, maior será a maximização do valor das empresas.

Assim, indo ao encontro da perspectiva de Flap e DeGraaf (1986), demonstrou-se que o capital do capital social realmente importa, pois os recursos fornecidos pelos alters são recursos potenciais que podem ser usados em benefício de uma firma individualmente. Entretanto, considerando a heterogeneidade das relações, verificou-se que as lacunas estruturais são meios de obter informações e recursos diversos, possibilitando uma maior vantagem competitiva pelas firmas, entre elas maior valor de mercado. Vale destacar que, no nosso estudo, esses recursos disponíveis nas lacunas estruturais impactaram significativamente o valor de mercado, o que ressalta que os conselheiros, especialmente aqueles com maior acesso a diferentes empresas e grupos de negócios, podem contribuir para uma avaliação mais positiva da companhia.

\subsection{IMPLICAÇÕES TEÓRICAS E PRÁTICAS}

Os resultados empíricos desta pesquisa e as discussões teóricas aqui apresentadas, como capital social, governança corporativa, board interlocking e redes, possibilitaram o desdobramento de algumas implicações teóricas e práticas. O primeiro entendimento teórico diz respeito ao posicionamento do conselho de administração e aos efeitos dele no desempenho das empresas. Diversos estudos se utilizaram das medidas de posicionamento de um ator na rede para testar suas hipóteses ou perguntas de pesquisas, como os estudos sobre "redes mundos pequenos" desenvolvidos por Mendes-da-Silva (20II) e Lazzarini (2007), o trabalho de Pusser, Slaughter e Thomas (2006), que estudaram o interlocking entre conselhos de empresas e universidades americanas, ou a pesquisa de Rossoni e Machado-da-Silva (20I3), que analisaram a relação entre legitimidade e valor de mercado em companhias listadas do BM\&F Bovespa. Diferentemente desses estudos, este artigo traz evidência de que a posição do conselho de administração não influenciou o valor de mercado. Isso reforça os argumentos de 
Mizruchi et al. (2006) de que o efeito de relações entre organizações está imerso em contextos institucionais e históricos que os condicionam.

A segunda implicação teórica está relacionada ao conceito de board interlocking e governança corporativa. De acordo com Davis e Mizruchi (I999), os interlockings podem influir econômica e socialmente nas organizações. Davis (I996) identificou que, nos Estados Unidos, os interlockings fornecem influências sociais nas práticas de governança. Rossoni e Mendes-da-Silva (20II) identificaram que empresas com melhor qualidade de governança corporativa, reputação organizacional e legitimidade do conselho de administração possuem influência no risco de ações negociadas em bolsa. Assim, este estudo contribui para o campo de estudos de board interlocking e governança corporativa ao estudar a análise do capital social do conselho de administração, evidenciando, empiricamente, como o interlocking influenciou o valor de mercado destas empresas.

A terceira e última implicação teórica está ligada à análise dos recursos relacionais do conselho de administração e de seu efeito no desempenho. Finegold, Benson e Hecht (2007) encontraram evidências de que o capital social do conselho de administração garante recursos, aconselhamento, melhor monitoramento e melhor desempenho para empresas. Kim (2007) traz evidências empíricas de que o capital social dos conselheiros externos é positivamente associado ao valor da empresa, por meio do indicador de $Q$ de Tobin. Este estudo contribui para estas pesquisas ao avaliar os recursos relacionais do conselho de administração por meio do valor de mercado da rede de relações das empresas, inovando ao medir o capital social pelos recursos imersos na rede. Isso vai além da forma de medição de capital social empregada por outros estudos, como os de Kim (2007), que mediu o capital social por meio de filiações em conselhos de empresas e conselhos de universidades, e Stevenson e Radin (2009), que mediram o capital social por meio de surveys com chief executive officers (CEOs).

Em termos de implicações práticas, esta pesquisa traz informações para os gestores de fundos de investimentos, gestores de empresas listadas na BM\&F Bovespa e investidores autônomos, para que, por meio da análise dos componentes do conselho de administração das empresas e dos indicadores de desempenho do valor de mercado, possam avaliar estratégias para, no caso de gestores de fundos, buscar uma melhor composição para sua carteira de ações, a fim de aumentar a rentabilidade dos fundos geridos. No caso de gestores de empresas listadas na bolsa, contribui-se para melhorar a composição de seus conselhos, buscar interlocking com conselheiros de empresas, com um melhor valor de mercado. Para os investidores autônomos, por sua vez, recomenda-se levar em consideração os conselhos de administração na avaliação do ambiente, antes de decidir onde investir o capital. 


\subsection{RECOMENDAÇÕES PARA ESTUDOS FUTUROS}

A primeira recomendação para trabalhos futuros é analisar as empresas de capital aberto com um olhar sobre os outsiders, seguindo o conceito de Portes (I998), para identificar como eles são afetados em função dos interlocks do conselho de administração. Como a governança corporativa é um tema de grande interesse, recomenda-se um aprofundamento da avaliação do capital social nos diversos níveis de governança. Além disso, deve-se verificar como as características de cada nível de governança afetam as empresas. A rede de proprietários deve ser analisada também até o último nível da estrutura, ou seja, até os proprietários das pessoas físicas. Outra recomendação é uma análise profunda sobre o comportamento de organizações que, ao passarem por alguma dificuldade, buscam fortalecer seus conselhos de administração, trazendo conselheiros de maior notoriedade ou que participam de conselhos de empresas, com melhor valor de mercado, com o objetivo de dar uma credibilidade maior à sua organização em um curto prazo. Aconselha-se também uma análise sobre o comportamento de conselheiros independentes, os quais deixam as organizações quando elas apresentam alguma dificuldade, para que sua imagem não seja afetada pelos problemas apresentados pelas empresas.

Por fim, orienta-se o uso de métodos de regressões espaciais e métodos estatísticos relacionais mais recentes para avaliar o efeito individual de cada recurso relacional e não de seu valor agregado pela soma no nível da firma.

\section{SOCIAL CAPITAL OF THE BOARD OF DIRECTORS AND PERFORMANCE OF BRAZILIAN PUBLIC COMPANIES}

\section{ABSTRACT}

Purpose: We analyze the effect of social capital of the board of directors on the market value of Brazilian public companies listed in BM\&F Bovespa. Therefore, we use as an indicator of social capital, relational resources present in the direct, indirect and heterogeneous ties to the board.

Originality/gap/relevance/implications: Our pioneer study indicates that inconsistent results of studies evaluating board interlocking often occur because most studies only consider the centrality and the position of the companies in the network. In doing so, they disregard that relations may have greater or lower degree of social capital.

Key methodological aspects: Empirically evaluate the relationship between companies through the directors and managers that they share,. Using social network 
analysis, we identified which are the directand indirect relations, as well as the structural gaps of the companies. We also measured the capital represented by each relationship from the market value of these relationships, which composed our measurement of relational resources. Then, through econometric models, we investigated the effects of relational resources on market value.

Summary of key results: Our results showed that heterogeneous relational resources have stronger and more significant influence than available resources in the direct relations of the board, while available resources in indirect relations were not significant.

Key considerations/conclusions: We demonstrated that the social capital really matters, because the resources provided by the alters are potential resources that can be used for the benefit of a firm, individually, It was found that structural gaps are ways to get information and various resources, enabling greater competitive advantage.

\section{KEYWORDS}

Social capital. Administrative Council. Social network analysis. Performance. Capital markets.

\section{CAPITAL SOCIAL DEL CONSEJO DE ADMINISTRACIÓN Y DESEMPEÑO DE LAS EMPRESAS BRASILEÑAS DE CAPITAL ABIERTO}

\section{RESUMEN}

Objetivo: Se buscó analizar el efecto del capital social del Consejo de Administración (board) en el valor de mercado de las empresas listadas en la BMF\&F Bovespa, utilizando como indicador del capital social, los recursos relacionales presentes en los lazos directos, indirectos y heterogéneos del board.

Originalidad/laguna/relevancia/implicaciones: Pioneros, nuestro estudio indica que las inconsistencias de los resultados de los estudios que evalúan las redes de consejeros muchas veces se producen porque la mayoría de los estudios sólo consideran la centralidad y la posición de las empresas en la red. De esta manera no se tiene en cuenta que las relaciones pueden tener mayor o menor grado de capital social.

Principales aspectos metodológicos: Empíricamente se evaluaron las relaciones entre las empresas partiendo de los directores y consejeros que comparten. Utilizando análisis de redes sociales, se identificaron cuáles son las relaciones directas, 
indirectas y las lagunas estructurales de las empresas, también se midió el capital representado por cada relación a partir del valor de mercado de esas relaciones, componiendo nuestra medida de recursos relacionales. Luego, a través de modelos econométricos, se investigaron los efectos de los recursos relacionales en el valor de mercado.

Síntesis de los principales resultados: Nuestros resultados mostraron que los recursos relacionales heterogéneos tienen una influencia más fuerte y más importante que los recursos disponibles en las relaciones directas del board, mientras que los recursos disponibles en las relaciones indirectas no fueron significativos. Principales consideraciones/conclusiones: Se ha demostrado que el capital social realmente importa, porque los recursos proporcionados por los alters son recursos potenciales que pueden ser utilizados para el beneficio de una empresa individual, y teniendo en cuenta la heterogeneidad de las relaciones, se encontró que las lagunas estructurales son formas de obtener informaciones y recursos, lo que permite una mayor ventaja competitiva.

\section{palabras CLAVE}

Capital social. Consejo de administración. Análisis de redes sociales. Desempeño. Mercado de capitales.

\section{REFERÊNCIAS}

Aldrighi, D. M., \& Mazzer, R., Neto (2007). Evidências sobre as estruturas de propriedade de capital e de voto das empresas de capital aberto no Brasil. Revista Brasileira de Economia, 61(2), I29-I52. Bhagat, S., \& Bolton, B. (2008). Corporate governance and firm performance. Journal of Corporate Finance, 14(3), 257-273.

Black, B., \& Kim, W. (20I2). The effect of board structure on firm value: a multiple identification strategies approach using Korean data. Journal of Financial Economics, 104(I), 203-226.

Black, B. S., Carvalho, A. G., \& Gorga, E. (20I2). What matters and for which firms for corporate governance in emerging markets? Evidence from Brazil (and other BRIK countries). Journal of Corporate Finance, 18, 934-952.

Borgatti, S. P. (1997). Structural holes: unpacking Burt's redundancy measures. Connections, 20(I), 35-38.

Bourdieu, P. (I980). Le capital social: notes provisoires. Actes de La Recherche en Sciences Sociales, 31(I), 2-3.

Bozec, R., Dia, M., \& Bozec, Y. (20I0). Governance-performance relationship: a re-examination using technical efficiency measures. British Journal of Management, 21(3), 684-700.

Burt, R. S. (1992). Structural holes: the social structure of competition. Cambridge: Harvard University Press. 
Carvalhal-Da-Silva, A. L., \& Leal, R. P. C. (2005). Corporate governance index, firm valuation and performance in Brazil. Revista Brasileira de Finanças, 3(I), I-I8.

Chung, K. H., \& Pruitt, S. W. (I994). A simple approximation of Tobin's Q. Financial Management, 23(3), 70-74.

Davis, G. F. (1996). The significance of board interlocks for corporate governance. Corporate Governance, $4(6)$, I54-I59.

Davis, G. F., \& Mizruchi, M. S. (I999). The money center cannot hold: commercial banks in the U. S. System of Corporate Governance. Administrative Science Quarterly, 44(2), 215-239.

De Nooy, W., Mrvar, A., \& Batagelj, V. (2005). Exploratory social network analysis with Pajek. New York: Cambridge University Press.

Finegold, D., Benson, G. S., \& Hecht, D. (2007). Corporate boards and company performance: review of research in light of recent reforms. Corporate Governance: An International Review, 15(5), $865-878$.

Flap, H. D., \& Degraaf, N. D. (I986). Social capital and attained occupational-status. Netherlands Journal of Social Sciences, 22(2), I45-I6I.

Fracassi, C., \& Tate, G. (20I2). External networking and internal firm governance. The Journal of Finance, 67(I), I53-I94.

Freeman, L. C. (I979). Centrality in social networks: conceptual clarification. Social Networks, 1(3), 2I5-237.

Freeman, L. C., Borgatti, S. P., \& White, D. R. (I99I). Centrality in valued graphs: a measure of betweenness based on network flow. Social Networks, 13(2), I4I-I54.

Granovetter, M. S. (I973). The strength of weak ties. American Journal of Sociology, 78(6), I36I-1380. Greene, W. H. (2000). Econometric analysis (4th ed.). Upper Saddle River: Prentice-Hall.

Haniffa, R. M., \& Cooke, T. E. (2002). Culture, corporate governance and disclosure in Malaysian corporations. Abacus, 38(3), 317-349.

Hanneman, R. A., \& Riddle, M. (2005). Introduction to social network methods. Riverside: University of California.

He, J., \& Huang, Z. (20II). Board informal hierarchy and firm financial performance: exploring a tacit structure guiding boardroom interactions. Academy of Management Journal, 54(6), III9-II39.

Instituto Brasileiro de Governança Corporativa (2009). Código de melhores práticas de governança corporativa (4a ed.). São Paulo: IBGC.

Johnson, S. G., Schnatterly, K., \& Hill, A. D. (2013). Board composition beyond independence: social capital, human capital, and demographics. Journal of Management, 39(I), 232-262.

Kim, Y. (2005). Board network characteristics and firm performance in Korea. Corporate Governance, $13(6), 800-808$.

Kim, Y. (2007). The proportion and social capital of outside directors and their impacts on firm value: evidence from Korea. Corporate Governance, 15(6), II68-ıі76.

38 Lazzarini, S. G. (2007). Mudar tudo para não mudar nada: análise da dinâmica de redes de proprietários no Brasil como "Mundos Pequenos". RAE-Eletrônica, 6(I).

Lazzarini, S. G. (20II). Capitalismo de laços. Rio de Janeiro: Elsevier, 20 II.

Lin, N. (200I). Social capital: capital captured through social relations. In N. Lin. Social capital: a theory of social structure and action (pp. I9-28). Cambridge: Cambridge University Press. 
Mendes-da-Silva, W. (20II). Small worlds and board interlocking in Brazil: a longitudinal study of corporate networks, I997-2007. Revista Brasileira de Finanças, 9(4), 52I-528.

Mendes-da-Silva, W., Ferraz-Andrade, J. M., Famá, R., \& Maluf Filho, J. A. (2009). Disclosure via website corporativo: um exame de informações financeiras de governança no mercado brasileiro. Revista de Administração de Empresas, 49(2), 190-205.

Mendes-da-Silva, W., \& Onusic, L. M. (2OI4). Corporate e-disclosure determinants: evidence from the Brazilian market. International Journal of Disclosure and Governance, 11(I), 54-73.

Mendes-da-Silva, W., Rossoni, L., Martin, L., \& Martelanc, R. (2008). A influência das redes de relação corporativas no desempenho das empresas do novo mercado da Bovespa. Revista Brasileira de Finanças, 6(3), 337-358.

Mizruchi, M. S. (1996). What do interlocks do? An analysis, critique, and assessment of research on interlocking directores. Annual Review of Sociology, 22, 27I-298.

Mizruchi, M. S., Stearns, L. B., \& Marquis, C. (2006). The conditional nature of embeddedness: a study of borrowing by large U. S. firms, I973-I994. American Sociological Review, 71(2), 310-333. Nahapiet, J. (2008). The role of social capital in inter-organizational relationships. In S. Cropper, M. Ebers, C. Huxham \& P. S. Ring. The Oxford handbook of inter-organizational relations (pp. 580-606). Oxford: Oxford University Press.

Portes, A. (1998). Social capital: its origins and applications in modern sociology. Annual Review of Sociology, 24, I-24.

Procianoy, J. L., \& Verdi, R. S. (2009). Adesão aos novos mercados da Bovespa: novo mercado, nível I e nível 2 - determinantes e consequências. Revista Brasileira de Finanças, 7(I), I07-I36.

Pusser, B., Slaughter, S., \& Thomas, S. L. (2006). Playing the board game: an empirical analysis of university trustee and corporate board interlocks. The Journal of Higher Education, 77(5), 747-775.

Rossoni, L., \& Machado-da-Silva, C. L. (2010). Institucionalismo organizacional e práticas de governança corporativa. Revista de Administração Contemporânea, 14(ed. esp.), I73-198.

Rossoni, L., \& Mendes-da-Silva, W. (20II). Nos limites da legitimidade: uma análise do risco no mercado de capitais brasileiro. Anais do Encontro Nacional da Associação Nacional de Pós-Graduação e Pesquisa em Administração - 3Es - Encontro de Estudos de Estratégia, Porto Alegre, RS, Brasil, 5 .

Rossoni, L., \& Machado-da-Silva, C. L. (20I3). Legitimidade, governança corporativa e desempenho: análise das empresas da BM\&F Bovespa. Revista de Administração de Empresas, 53(3), 272-289. Santos, R. L., \& Silveira, A. M. (2007). Board Interlocking no Brasil: participação de conselheiros em múltiplas companhias e seu efeito sobre o valor das empresas. Revista Brasileira de Finanças, 5(2), I25-163.

Silveira, A. M., Barros, L. A. B. C., \& Famá, R. (2006). Atributos corporativos, qualidade da governança corporativa e valor das companhias abertas no Brasil. Revista Brasileira de Finanças, 4(I), I-3o. Silveira, A. D. M., Leal, R. P. C., Carvalhal-Da-Silva, A. L., \& Barros, L. A. B. D. C. (20IO). Endogeneity of Brazilian corporate governance quality determinants. Corporate Governance, 10(2), I9I-202. Stevenson, W. B., \& Radin, R. F. (2009). Social capital and social influence on the board of directors. Journal of Management Studies, 46(I), I6-44.

Uzzi, B., \& Spiro, J. (2005). Collaboration and creativity: the small world problem. American Journal of Sociology, 111(2), 447-504.

Weber, C., Davis, G. F., \& Lounsbury, M. (2009). Policy as myth and ceremony? The global spread of stock exchanges, I980-2005. Academy of Management Journal, 52(6), I3I9-I347. 